\title{
How can LCA include prospective elements to assess emerging technologies and system transitions? The 76th LCA Discussion Forum on Life Cycle Assessment, 19 November 2020
}

\author{
Lugas Raka Adrianto ${ }^{1}$ Mitchell K. van der Hulst ${ }^{2,3}$. Janot P. Tokaya ${ }^{3} \cdot$ Rickard Arvidsson $^{4}$. Carlos F. Blanco ${ }^{5}$. \\ Carla Caldeira $^{6}$. Gonzalo Guillén-Gonsálbez $z^{7}$ Serenella Sala ${ }^{6} \cdot$ Bernhard Steubing $^{5} \cdot$ Matthias Buyle $^{8,9}$. \\ Mohamad Kaddoura $^{10}$. Nicolas H. Navarre ${ }^{5}$. Julien Pedneault ${ }^{10}$. Massimo Pizzol ${ }^{11}$. Beatrice Salieri ${ }^{12}$. \\ Toon van Harmelen ${ }^{3}$. Mara Hauck ${ }^{3}$ (1)
}

Received: 22 April 2021 / Accepted: 30 May 2021 / Published online: 20 July 2021

(c) The Author(s), under exclusive licence to Springer-Verlag GmbH Germany, part of Springer Nature 2021

\begin{abstract}
This paper summarizes the 76th LCA Discussion Forum end its main findings. Main issues when addressing emerging technologies identified were: the lack of primary data, the need for (shared) future background scenarios and (guidlines for) a common methodology. The following recommendations have been derived by the organizers: 1) Specific foreground inventories are always tailor-made, but consistency can be improved through lists of mandatory considerations. 2) Continue sharing (future) technology data and proxy processes, that can be readily replicated to new studies and assist in developing inventories. 3) Streamline and unify the process of including scenarios for background systems. New approaches may provide first important solutions to efficiently include consistent future scenarios in prospective LCA.
\end{abstract}

Keywords LCA discussion forum $\cdot$ Emerging technologies, $\cdot$ Prospective LCA $\cdot$ Transitions

Communicated by Matthias Finkbeiner

Poll methods/results, extended descriptions of presenters' talks, and full list of references are accessible in the accompanying Supporting Information. More materials, including all presentations from the 76th LCA Discussion Forum, are available for download at http://www.lcaforum.ch, while the video recordings of all sessions can be watched online at http://www. video.ethz.ch/events/lca/2020/autumn/76th.html.

Mara Hauck

mara.hauck@tno.nl

1 Institute of Environmental Engineering, Chair of Ecological Systems Design, ETH Zürich, John-von-Neumann-Weg 9, 8093 Zurich, Switzerland

2 Department of Environmental Science, Radboud University, Heyendaalseweg 135, 6525AJ Nijmegen, the Netherlands

3 Department of Climate, Air and Sustainability, TNO, Princetonlaan 6, 3584CB Utrecht, the Netherlands

4 Environmental Systems Analysis, Chalmers University of Technology, Vera Sandbergs Allé 8, 41296 Gothenburg, Sweden

5 Institute of Environmental Sciences (CML), Leiden University, Einsteinweg 2, 2333CC Leiden, the Netherlands

6 European Commission-Joint Research Centre, Via Enrico Fermi 2749, 21027 Ispra, Italy

\section{Introduction}

The 76th Life Cycle Assessment Discussion Forum on November 19, 2020, titled "The use of LCA as a development tool for emerging technologies / How to deal with forecasts in LCA?" set out to identify which tools and

7 Institute for Chemical and Bioengineering, Department of Chemistry and Applied Biosciences, ETH Zürich, Vladimir-Prelog-Weg 1, 8093 Zurich, Switzerland

8 Energy and Materials in Infrastructure and Buildings (EMIB), Applied Engineering, University of Antwerp, Groenenborgerlaan 171, 2020 Antwerp, Belgium

9 Unit Sustainable Materials Management, Flemish Institute for Technological Research (VITO), $2400 \mathrm{Mol}$, Belgium

10 Department of Industrial Engineering, CIRAIG, École Polytechnique de Montréal, Centre-ville, P.O. Box 6079, SuccMontreal, QC H3C 3A7, Canada

11 Department of Planning, Aalborg University, Rendsburggade 14, 9000 Aalborg, Denmark

12 Swiss Federal Laboratories for Materials Science and Technology (Empa), Technology and Society Lab (TSL), Lerchenfeldstrasse 5, 9014 St. Gallen, Switzerland 
approaches are currently used, what kind of challenges are associated with them, and what further aspects can and need to be considered.

\section{Methods to evaluate environmental impacts of new technologies}

Assessments of emerging technologies in their embryonic state, produced at laboratory scale with current surrounding systems, might poorly reflect the future environmental impacts of such technologies. Rickard Arvidsson (Associate Professor at Chalmers University, Sweden) espoused that approaches such as prospective and ex-ante LCA attempt to resolve this by modelling emerging technologies at a future point in time when the technologies are mature and produced at large scale. Gonzallo Guillén-Gosálbez (Associate Professor at ETH Zürich, Switzerland) showed that the integration of mathematical tools with LCA provides a powerful framework to address optimization challenges for a more sustainable industry, where substantial environmental savings can be attained at a marginal increase in cost. Carlos Blanco (PhD student at CML, Leiden University, the Netherlands) made a case for the use of probabilistic scenario modelling of technological choices in combination with global sensitivity analysis to handle the numerous degrees of freedom that arise in LCA models of emerging technologies. This approach filters out design choices that cannot noticeably shift impact score distributions despite their uncertainties.

We learned from this section that prospective LCA is used to address three levels of change: (emerging) technologies themselves, production processes and surrounding production systems. For enabling sustainable transitions, we should also consider planetary boundaries and interactions between different systems (e.g. energy production and base materials). Tools are already available, like optimization algorithms, probabilistic use of scenarios and global sensitivity analysis. At their current state, these are largely academic tools as they are in a pioneering stage, relatively data- and time-intensive.

\subsection{LCA for emerging technologies: case study methods, policy needs and software for application}

Mitchell van der Hulst (PhD student at Radboud University and TNO, the Netherlands) presented an applicationcentred systematic approach for the prospective LCA process that includes consecutive steps from LCI changes in lab/pilot to industrial scale and industrial learning and background changes. Applicability of the approach was demonstrated with a case study of an emerging PV technology. Lugas Raka Adrianto (PhD student at ETH Zürich, Switzerland) presented a case study of multiple valorization pathways of mine waste that involve prospective LCA in the whole value chain. Technological mapping was used to include different pathways and technology readiness levels. In the context of applying prospective LCA as support tools for policy designs, Serenella Sala (Scientific officer at EU Commission Joint Research Centre, Italy) emphasized that innovation plays a key role to help reaching current EU policy ambitions goals, such as the European Green Deal. Bernhard Steubing (Assistant Professor at CML, Leiden University, the Netherlands) presented how background scenarios derived, e.g. from Integrated Assessment Models (IAMs), can be included more easily in LCA by making use of a superstructure database in combination with scenario difference files in Activity Browser (Steubing et al. 2020).

From these insights we draw the following lessons:

- The need for and usefulness of prospective LCA in helping to choose the most sustainable technology for transitions is confirmed from a policy perspective. However, it is also highlighted that comprehensibility of its results for decisionmakers is a precondition for successful application.

- Technology mapping is a tool that can help understand the contributions of technologies following a realistic time path (depending on technology readiness levels (TRLs) and other restrictions to availability).

- Including the impacts of technologies at different TRLs can be fed by diverse approaches to systematically include process changes, size scaling, potential synergies, industrial learning (in the foreground inventory) and external developments (in the background).

- The superstructure databases and software such as Activity Browser allows combining these foreground and background scenarios in a way that can be included by many practitioners in their LCA studies.

\subsection{Short presentations}

Six short presentations were held with various topics in prospective LCA, ranging from framework developments by Nicolas Navarre (PhD student at CML, Leiden University, the Netherlands) and Beatrice Salieri (Scientist at Empa, Switzerland), case studies by Matthias Buyle (Postdoc at University of Antwerp and VITO, Belgium), uncertainties handling by Massimo Pizzol (Associate Professor at Aalborg University, Denmark) to linking of LCA with integrated assessment models and scenario projections by Mohamad Kaddoura and Julien Pedneault (PhD students at Polytechnique Montréal, Canada). 
Overall, the open floor presentations confirmed that more and more researchers are concerned with including scenarios of change in the technology inventory (foreground) as well as the surrounding system (background). However, often the focus is on one of the two. For the foreground inventory, upscaling approaches and detailed modelling of subprocesses and their combinations are the dominant approaches. Background scenarios can be derived using insights from the IAM community, with conditions set by the Shared Socioeconomic Pathways (SSP) and Representative Concentration Pathway (RCP) frameworks. Next to that, increasing attention is paid to appropriately including uncertainty assessments and lessons from other approaches such as risk assessment.

\section{Panel discussions}

A group of seven experts from academia and industry was invited to share their insights in two interactive discussion rounds.

\subsection{Theme 1: Prospective models and potential solutions}

Thomas Gibon (LIST, Luxembourg) gave a few brief examples of integrated energy scenario modelling (ESM) in LCA. The framework proposed in the presentation has been put forward as a structured way to include large-scale systemic changes, with a definition of the various levels of ESM-LCA integration. One expected output from this work is described by Toon van Harmelen (TNO, the Netherlands), which is a prognostic LCA with background scenarios available in databases. Roland Hischier (Empa, Switzerland) also emphasized the importance of such forward-looking databases that are equipped with technology upscaling information. Taking into account these needs, Romain Sacchi (PSI, Switzerland) also put forward as a solution to align the life cycle inventories in standard databases (i.e. Ecoinvent) and integrated assessment models. These databases could then become a common platform for conducting prospective LCAs, solving the disparity in approaches, and reducing the workload for this part of conducting a prospective LCA.

\subsection{Theme 2: Challenges for applications and decision-making}

When it comes to bringing the prospective studies into business and policymaking cases, both Bruno van Parys (Solvay, Belgium) and Serenella Sala (JRC, Italy) recognize the strengths of LCA. However, the former stressed that studies with more than three scenarios are outside of the corporate considerations in most situations as the interpretation of results becomes too challenging. Therefore, constructing future storylines that can be described in a reasonable number of scenarios and time horizons is key.

\section{Synthesis}

Three main challenges for LCAs for emerging technologies have been discussed throughout the forum:

\subsection{The lack of primary data}

Presented approaches to estimate full-scale LCIs for which data are not yet available were inclusion of process changes by interaction with technology developers, economies of scale when using larger equipment, modelling of plant synergies, thermodynamic process simulations, empirical design rules and proxy technology transfer. While upscaling relations were mentioned several times, industrial learning was only included in one study.

\subsection{The need for (shared) future background scenarios}

The most common approach for adapted background scenarios was making use of scenarios from IAMs which are set to conditions for various combinations of SSPs. In most cases, a soft link was performed where, for instance, projected efficiency gains or technology distributions in future energy mixes were transferred into LCI databases. A combination of new approaches by Mendoza-Beltran et al. (2020) and Joyce and Björklund (2021) and the implementation possibilities via superstructure databases could be a way to efficiently include scenarios in a more standardized way.

\section{3 (Guidelines for) a common methodology}

Although there are many joint opportunities, methodology development has not yet converged to a level where it is reflected in norms and guidelines. However, first approaches are visible that give the various approaches a common framework from which some or several steps can be included in a prospective LCA, such as the stepwise approach from van der Hulst et al. (2020).

Since we are at the start of the development of prospective LCA, it is too early to formalize data formats, applications and methodologies in particular standards. However, the organizers of this discussion forum feel some steps can already be taken and included in everyday LCA. We, therefore, recommend increasing collaboration on the following approaches: 
- Specific foreground inventories are always tailor-made, but consistency can be improved through lists of mandatory considerations.

- Continue sharing (future) technology data and proxy processes, which can be readily replicated to new studies and assist in developing inventories.

- Streamline and unify the process of including scenarios for background systems. New approaches may provide first important solutions to efficiently include consistent future scenarios in prospective LCA.

We hope that the recommendations can be advanced in a concerted effort by software and database developers, LCA practitioners, the scientific community and guideline and harmonization initiatives.

Supplementary information The online version contains supplementary material available at https://doi.org/10.1007/s11367-021-01934-w.

Acknowledgements The organizers of the Discussion Forum would like to thank the sponsors (Carbotech, ETH Zurich, treeze Ltd. and Empa), the advisory board, the technical team and, most importantly, the speakers, the panellists (listed in the previous sections) and participants for their contribution to the success of the event. Lugas Raka Adrianto and Matthias Buyle are grateful for the funding provided by the EU Framework Programme for Research and Innovation Horizon 2020 under Grant Agreement No. 812580 'SULTAN' and No. 730471

'CHROMIC', respectively.

\section{References}

Joyce PJ, Björklund A (2021) Futura: a new tool for transparent and shareable scenario analysis in prospective life cycle assessment. J Ind Ecol. https://doi.org/10.1111/jiec.13115

Mendoza Beltran A, Cox B, Mutel C, van Vuuren DP, Font Vivanco D, Deetman S, Edelenbosch OY, Guinée J, Tukker A (2020) When the background matters: using scenarios from integrated assessment models in prospective life cycle assessment. J Ind Ecol 24:64-79. https://doi.org/10.1111/jiec.12825

Steubing B, de Koning D, Haas A, Mutel CL (2020) The Activity Browser - an open source LCA software building on top of the brightway framework. Softw Impacts 3:100012. https://doi.org/ 10.1016/j.simpa.2019.100012

van der Hulst MK, Huijbregts MAJ, van Loon N, Theelen M, Kootstra L, Bergesen JD, Hauck M (2020) A systematic approach to assess the environmental impact of emerging technologies: a case study for the GHG footprint of CIGS solar photovoltaic laminate. J Ind Ecol 24:1234-1249. https://doi.org/10.1111/jiec.13027

Publisher's Note Springer Nature remains neutral with regard to jurisdictional claims in published maps and institutional affiliations. 